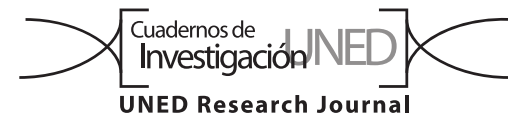

\title{
Participación ciudadana: retos para una ciudadanía activa ante el cambio climático
}

\author{
Carolina Álvarez-Vergnani \\ FLACSO-Sede Académica de Costa Rica, caroalvarez@flacso.or.cr, caroav@gmail.com \\ Recibido 14-VIII-2018 • Corregido 20-XII-2018 • Aceptado 14-I-2019
}

\begin{abstract}
Citizen participation: challenges for an active citizenship facing climate change. Democracy is measured by the degree of citizen participation in decision-making processes, not only those that are procedural, but also those that require people own action. Despite the importance of participatory processes, as well as the dedication of time and economic resources provided by States to the formulation of public policy related to addressing climate change, in many Latin American populations, they perceive that the processes are not suitable, sufficient or efficient. While it is true that, from the Government, these spaces should be promoted and actions taken to strengthen citizen skills, as well as institutional capacities and the regulatory body to achieve participatory processes of weight, as citizenship we must question whether we are really demanding these spaces of the best way and in the cases where they exist, if we are making a correct, profitable and strategic use. The information, the issuance of assertive criteria, and an active intervention, are levels of participation that ideally should always be present, strengthening both the structures and the actions for governance, in search of the common good. The most evolved forms of conflict resolution should be clear that democracy is not equal to the unanimity, and that these processes rather seek common ground in pluralism. A weakening of participatory processes can cause a weakening of democracy, poor management of public goods and services, and therefore the privatization of the State. This implies that as citizens we must learn to inform ourselves, to be clear about our interests, to dialogue and negotiate in search of agreements, and to consider, therefore, the diversity of opinions in the process.
\end{abstract}

Key words: citizen participation, democracy, citizenship, governance, dialogue, negotiation, climate change.
RESUMEN: La democracia se mide por el grado de participación de la ciudadanía en los procesos de toma de decisión, no sólo aquellos que son procedimentales, sino en los que se requiere la acción propia de las personas. A pesar de la importancia de los procesos participativos, así como la dedicación de tiempo y recursos económicos que brindan los Estados a la formulación de política pública relacionada a la atención del cambio climático, en muchas poblaciones latinoamericanas, estas perciben que los procesos no son aptos, suficientes o eficientes. Si bien es cierto que, desde el Gobierno, deben promoverse estos espacios y ejecutarse acciones que fortalezcan las habilidades ciudadanas, así como las capacidades institucionales y el cuerpo normativo para lograr procesos participativos de peso, como ciudadanía debemos cuestionarnos si realmente estamos demandando estos espacios de la mejor manera y en los casos donde existen, si estamos haciendo un uso correcto, provechoso y estratégico. La información, la emisión de criterios asertivos y la intervención activa, son niveles de participación que idealmente siempre deberían estar presentes, fortaleciendo tanto las estructuras, como el accionar para la gobernanza, en búsqueda del bien común. Las formas más evolucionadas de solución de conflictos deben tener claro que la democracia no es igual a la unanimidad, y que estos procesos más bien buscan puntos en común dentro del pluralismo. Un debilitamiento en los procesos participativos puede provocar un debilitamiento de la democracia, un mal manejo de los bienes y servicios públicos, y por ende la "privatización del Estado". Esto implica que, como ciudadanía debemos aprender a informarnos, a tener claros nuestros intereses, a dialogar y negociar en búsqueda de acuerdos, y a considerar, por tanto, a las diversidades de opiniones en el proceso.

Palabras claves: participación ciudadana, democracia, ciudadanía, gobernanza, diálogo, negociación, cambio climático.

\section{Participación ciudadana: definición}

Según la Declaratoria Universal de los Derechos Humanos, la participación ciudadana se define como la intervención de la ciudadanía en los procesos de toma de decisiones (Comisión de Derechos Humanos, 1948), dejando de ser una concesión y pasando a ser un derecho legítimo. Dicho de otra forma, más expresa, la participación ciudadana representa un elemento fundamental de un sistema democrático, inclusivo y representativo, en el que se incluyen un conjunto de acciones, formales o informales, a las que las personas acudimos para ser parte del proceso deliberativo y de toma de decisiones.

Inicialmente el concepto de participación ciudadana se restringía al voto, pero posteriormente fue transformándose, incluyendo otras formas de acción como el contacto con cargos políticos, la participación en 
campaña electoral, la cooperación en comunidades, la protesta, entre otros (Anduiza \& Bosch, 2004). Todas estas formas, relacionadas a los derechos, bienes y servicios de carácter público, que pueden estar o no asociadas al Estado o al sector privado.

De esta forma la participación ciudadana puede darse en representación de un conjunto específico de personas o a título personal, sin la necesidad de formar parte de un grupo político, de la Administración Pública o de un colectivo.

\section{¿Está en crisis la participación ciudadana?}

Existen muchas teorías que hipotetizan si nos encontramos o no frente a una crisis de ciudadanía. Desde aquellas que se refieren a una crisis generalizada en todas las democracias postindustriales (Putnam \& Goss, 2002), a aquellas que consideran que la crisis es más bien de carácter "participativo o representativo", pero no "democrático" (Norris, 1999a, 2002). La primera teoría es defendida mayormente por Putman, basándose en el caso estadounidense ocurrido a partir de los años sesenta, como escenario para anticipar lo que sucede en otras latitudes en un mundo globalizado. Para esto, acude a que los cambios sociales, políticos y tecnológicos, dados a partir de esa década, debilitan los mecanismos tradicionales de producción de capital social y, por tanto, la participación de la ciudadanía en los asuntos públicos, volviendo a las nuevas cohortes más apáticas en asuntos de participación.

En contraposición a esta idea, Norris (2002) plantea la reinvención del activismo político, haciendo mención de que esa misma globalización ha generado mayores niveles de educación que propician una evolución y diversificación de las formas, objetivos y estructuras de participación, por lo que actualmente nos podríamos estar enfrentando más bien, a un cambio generacional en cuanto a las pautas y los niveles de participación y no, necesariamente a una apatía ciudadana.

Como un tercer planteamiento, existen analistas como Morales (2005) que se refieren a este asunto diciendo que ambas teorías se neutralizan y más bien son complementarias, e inclusive hay planteamientos que apoyan la teoría de que las nuevas cohortes son más participativas (Welzel, Inglehart \& Deutsch, 2005). Ambas conclusiones radican en que actualmente se utilizan nuevas formas de participación que no son convencionales y que, por lo tanto, en muchas latitudes no se consideran formales. Estas nuevas pautas pueden darse con un grado variable de intensidad y frecuencia, y con distintos grados de iniciativa y esfuerzo (Anduiza \& Bosch, 2004), sin embargo, siempre estarán acompañadas de los procesos formales de participación.

Según Anduiza y Bosch (2004), paralelo a estas teorías acerca de si nos encontramos o no en una crisis de participación, se pueden contemplar también distintas tipologías que diferencian el nivel de acción de los y las ciudadanas. Entre estas se mencionan las propuestas presentadas por Milbrath (1977), referentes a tres niveles de participación política, donde aproximadamente un $30 \%$ de las personas eran apáticos, un 60\% eran espectadores que participan esporádicamente y una minoría inferior al $10 \%$ se consideraban como los que realmente participaban. Otra propuesta es la de Barnes y Kaase (1979), que basaban su división en cinco categorías: personas inactivas, conformistas, contestatarias, reformistas y activistas, incluyendo así nuevas formas de participación distintas al voto e incorporando distintos grados de intensidad e incidencia. La percepción de que la política es importante y que la propia actividad política puede incidir en el funcionamiento del sistema político, son actitudes que incentivan a la participación. Esto sumado a las actitudes cognitivas, afectivas y valorativas del individuo, la eficacia interna (habilidades y capacidades que el individuo reconoce en sí mismo como actor político) y la eficacia externa (percepción de que el sistema político es sensible a sus demandas) es lo que podría definir si una persona participa, cómo y qué tanto.

A continuación, el escrito describe algunos puntos que refuerzan o debilitan estas teorías, vistos desde la perspectiva de las instituciones públicas, la propia ciudadanía y la gobernanza que generan entre ambas. Posteriormente se mencionan opciones para un mejor abordaje y posibilidades dentro de la agenda climática.

\section{Los retos de la institucionalidad pública}

Las incapacidades institucionales, incluyendo recursos presupuestarios, humanos, técnicos e inclusive, los niveles de sensibilización, conocimiento y/o interés político, para realizar procesos participativos formales por parte del Estado, son ampliamente conocidas y criticadas a lo largo de Latinoamérica. Ejemplo de esto son los resultados generales de una encuesta aplicada en la región, por diversas organizaciones de sociedad civil y expertos en materia de cambio climático, cuyo fin consistió en medir los niveles de ambición, desde la perspectiva de actores no gubernamentales, en la formulación de las Contribuciones Previstas y Determinadas a Nivel Nacional (Intended Nationally Determined Contributions, INDC) en el año 2015. 


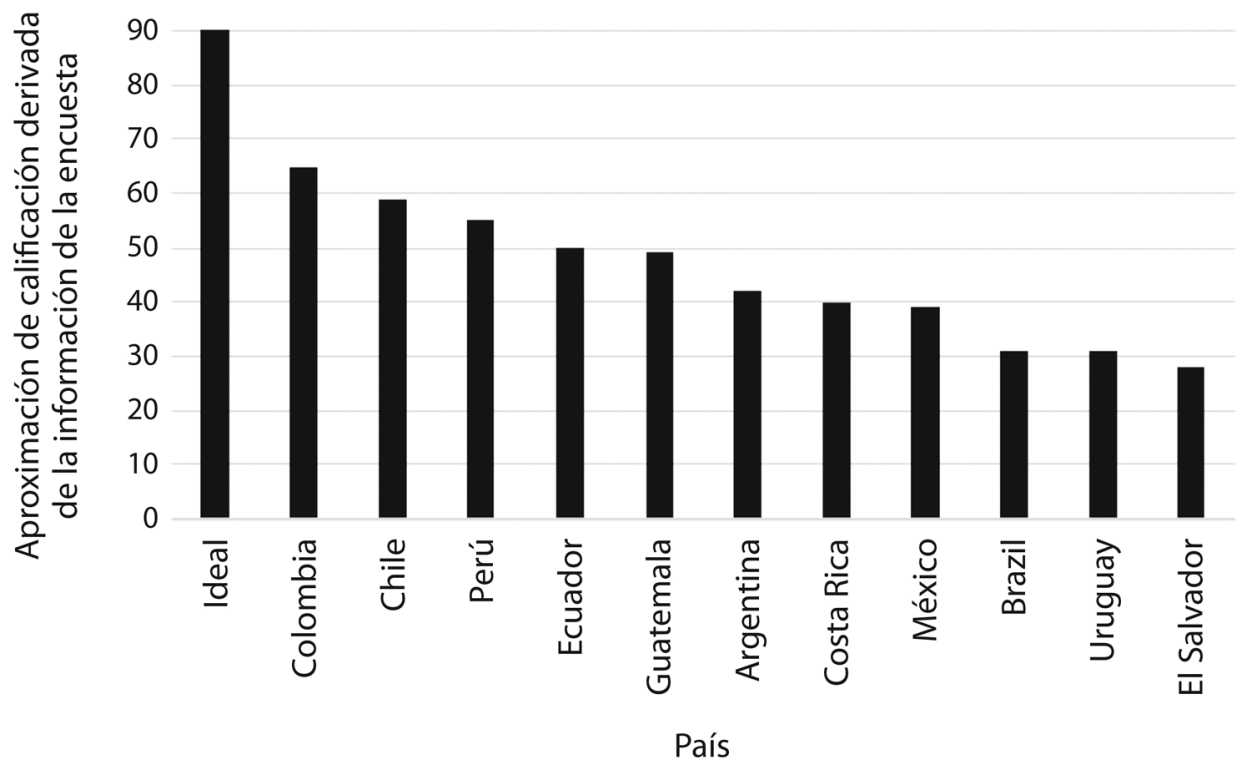

Fig. 1. Aproximación de la calificación general sobre la participación ciudadana en los procesos de formulación de los INDC en Latinoamérica. Basado en Guzmán y Castillo (2016).

La encuesta, aplicada a 11 países de Latinoamérica (Argentina, Brasil, Chile, Colombia, Costa Rica, Ecuador, El Salvador, Guatemala, México, Perú, Uruguay) se basó en ocho criterios de análisis: medidas de mitigación, adaptación, financiamiento, transferencia de tecnología y creación de capacidades, acceso a la información, convocatoria, consulta y participación, y gobernanza. Como se puede observar en la Fig. 1, el resultado más alto lo obtuvieron Colombia y Chile, los cuales alcanzaron niveles cercanos a las dos terceras partes de la puntuación de 90, considerada como ideal (Guzmán \& Castillo, 2016). Estos resultados reflejan, entre otras cosas, que las partes consultadas perciben que las INDC no representaron una visión integrada del país y no tomaron en cuenta las necesidades o propuestas de los sectores más vulnerables de estos, a pesar de contar con un proceso formal de participación, e inclusive estar presente dentro de su marco normativo de mayor jerarquía. Otro ejemplo en esta misma línea es demostrado por el caso costarricense, donde la puntuación alcanzada en este proceso fue menor a la mitad del puntaje máximo, a pesar de que el artículo 9 de su Constitución Política, modificado en el año 2003, hace alusión a que "El Gobierno de la República es popular, representativo, participativo, alternativo y responsable" $y$ su artículo primero, modificado en el año 2015, resalta a esta República como "democrática, libre, independiente, multiétnica y pluricultural", reconociendo así la diversidad de los actores involucrados.
Posterior a las formulaciones de las INDC, donde se realizaron estos procesos participativos, se han generado distintos criterios para medir la aplicación por parte de los Gobiernos, del principio 10 de la Declaración de Río de 1992, así como los compromisos asumidos por las partes en la Convención Marco de las Naciones Unidas de Cambio Climático (CMNUCC), referentes a la participación de la sociedad civil en torno a temáticas relacionadas a este fenómeno. Entre estos criterios se pueden mencionar los provenientes de organismos internacionales, como el documento elaborado por la Comisión Económica de Naciones Unidas para América Latina y el Caribe (CEPAL), denominado "Acceso a la información, la participación y la justicia en asuntos ambientales en América Latina y el Caribe: hacia el logro de la Agenda 2030 para el Desarrollo Sostenible" (CEPAL, 2018); así como los planteados desde la propia sociedad civil, caso de la Iniciativa de Acceso (The Acces Iniciative, TAl) o la crítica de activistas climáticos como Granados-Solís (2017), referente a la transparencia.

Como se puede ver en los resultados del análisis (Fig. 2), de 24 mecanismos de coordinación interinstitucional para una política climática efectiva en Latinoamérica y el Caribe, la inclusión de la totalidad de distintos actores en el proceso (autoridades sectoriales, gobiernos subnacionales, sector privado, Organizaciones No Gubernamentales (ONG) y universidades/centros de investigación), equivale únicamente al $28 \%$ de los casos, es 


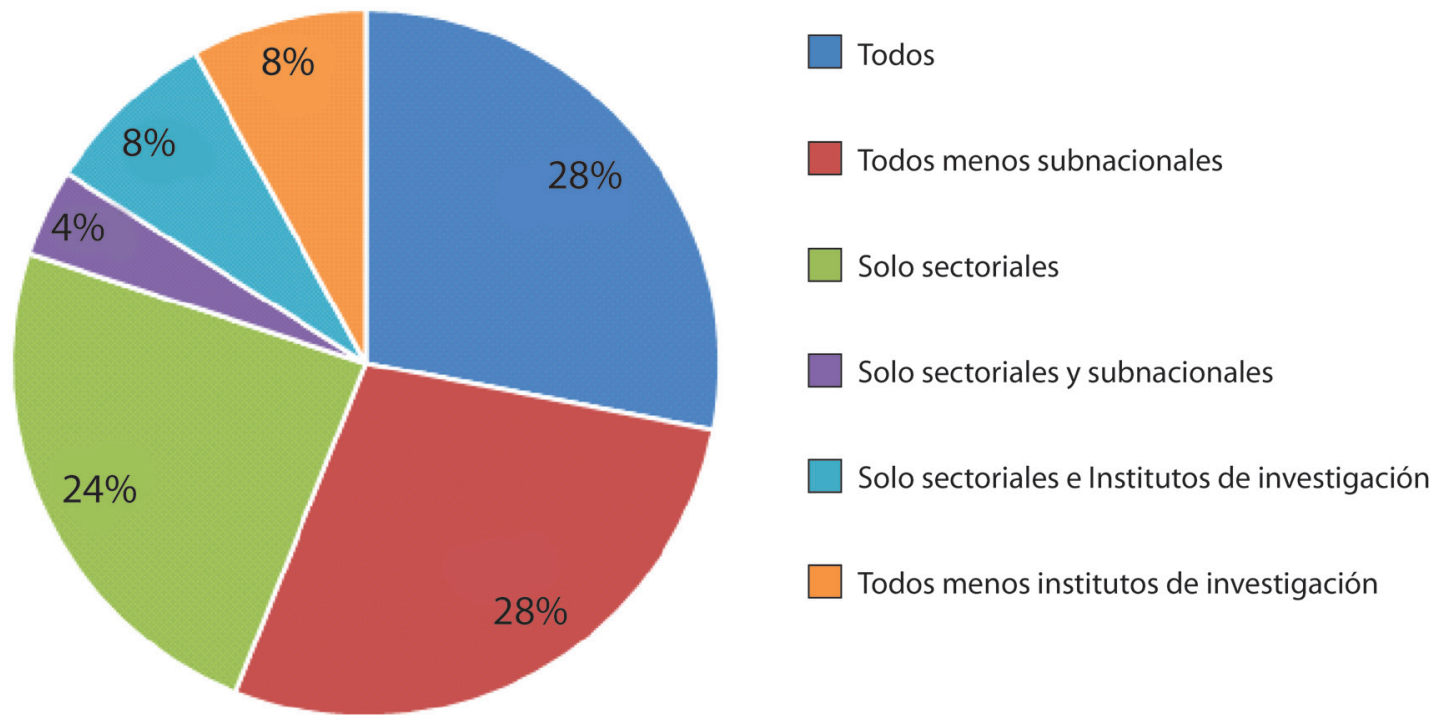

Fig. 2. Representación de actores dentro de los mecanismos de coordinación interinstitucional en 24 países de Latinoamérica. Basado en Plataforma LEDS LAC et al. (2017).

decir siete de veinticuatro países del estudio (Plataforma LEDS LAC et al., 2017).

Lo anterior deja claro por un lado, que los países sí están realizando importantes cambios a nivel de normativa en torno a la participación y su consecuente operativización. Sin embargo, a pesar de que los Gobiernos han comenzado a internalizar la importancia de la inclusión de los distintos actores en los procesos de toma de decisión, para poder contar con una visión completa, informada e inclusive avalada por las partes que se gobiernan, aún quedan presentes rezagos que producen malestar en la ciudadanía.

Muchos de estos rezagos podrían tener sus causas en el hecho de que los funcionarios públicos están obligados a cumplir los deberes que la ley impone, pero no pueden arrogarse facultades más allá que las que ya le están normadas, corriendo el riesgo de recibir acciones penales públicas, que incluso pueden tener una consecuente responsabilidad personal en muchos países (Caso de Costa Rica, artículo 11 de la Constitución Pública). Desde una perspectiva, este hecho podría disminuir la proactividad de los y las funcionarias, de ejecutar acciones innovadoras; pero también podría ser visto como una forma de presionar al aparato institucional para que ejecute las acciones para las que fue dispuesto, a través de la evaluación y rendición de cuentas de su personal.

Bajo la línea de los rezagos institucionales, Norris (1999b) realiza cuatro afirmaciones en cuanto a cómo la erosión de la confianza en las instituciones de democracia representativa, es decir, aquella en la que "se identifica la voluntad de los representantes del pueblo soberano con la de sus representantes electos" (Hernández, 2002) puede alterar los niveles y las formas de participación de la ciudadanía. La primera de ellas, relacionada a cómo estos fallos institucionales pueden disuadir a la ciudadanía de la participación electoral, el activismo político y/o el compromiso cívico; segundo, cómo esa alienación producida con el régimen formal puede aumentar las formas de protesta y activismo no convencional; tercero, cómo cuando existe confianza en la institucionalidad, se fomenta el cumplimiento voluntario de la ley y con eso la capacidad de los gobiernos para aprobar e implementar una legislación efectiva, sin la necesidad de coacción; $y$, por último, cómo las crecientes tensiones entre el ideal y la realidad pueden obstaculizar el proceso de consolidación en las democracias más nuevas.

En este sentido es importante resaltar, el reto que tienen los Estados y su institucionalidad pública, de mantener un balance entre la democracia representativa y la democracia participativa, entendiendo esta última como aquella en la que la ciudadanía escoge en conjunto sus leyes y toma sus propias decisiones (Hernández, 2002). En referencia a esto, debe decirse que es importante contar con una democracia participativa que permita a la ciudadanía desligarse de la toma final de decisiones, cuyos temas no son manejados por la mayoría. Sin embargo, hay otros temas en los que, debido al impacto que recibe, es necesario contar con una vinculación directa, tales como la gestión del riesgo local, la adaptación climática 
de sus medios de vida, la mitigación de los gases de efecto invernadero producto de sus propias actividades y la demanda de la rendición de cuentas del aparato institucional que debiera respaldarle.

Aparte de estos retos institucionales, pocas veces nos ponemos a pensar si la ciudadanía, a su vez, está respondiendo de la mejor manera a las oportunidades que presentan los Gobiernos al dar estos espacios, y en los casos en los que no se dan, analizar si la ciudadanía los está sabiendo demandar, haciendo uso de sus facultades o si ella misma se está abriendo espacio en los casos donde este espacio es reducido.

\section{Los retos de la ciudadanía}

A pesar de los avances por parte de algunos Estados, tanto en la incorporación de espacios para la participación, como en la mejora de la calidad de muchos de sus procesos formales, en torno a temáticas relacionadas al cambio climático, cuando se habla del aporte y de las responsabilidades de la ciudadanía, el tema se aborda con menor frecuencia y en muchos casos se hace desde una perspectiva bastante negativa.

Dentro de los puntos que se consideran básicos para ofrecer mejoras a los procesos participativos de cualquier índole, en búsqueda del buen funcionamiento del Estado y por tanto del bien común -incluyendo aquellas variaciones que puedan surgir como producto de los cambios en los patrones del clima-, se pueden generalizar tres niveles básicos de participación desde la perspectiva ciudadana. El primer nivel correspondería a estar informados e informadas ante cualquier proceso, sin importar su magnitud, profundidad o complejidad. Seguido de este, el segundo nivel consistiría entonces en ser parte de algún proceso consultivo de cara a la toma de decisiones, teniendo una participación aún un poco pasiva. Por último, un tercer nivel, con mayor incidencia, incluiría una participación activa dentro de las estructuras propias de gobernanza, entendiéndose esta como la interrelación de las estructuras formales e informales, así como de los procedimientos, procesos y las formas de hacer cumplir las reglas.

Para que el Derecho Humano a la participación ciudadana se cumpla de forma responsable, cualquiera de estos tres niveles de participación, tienen a su vez tres requerimientos básicos que recaen finalmente también en la ciudadanía y no únicamente en las instituciones del Estado. El primero de ellos se refiere al interés y la seriedad con la que se dé la participación, la cual dependerá en gran medida del grado de sensibilidad acerca de cómo el asunto afecta al colectivo, así como del conocimiento en cuanto al grado y las formas en las que lo hace. El segundo punto es entender qué significa participar y hasta dónde llega nuestra injerencia en cada uno de los procesos en los que nos podemos ver inmersos. Importante recalcar que esta participación se da durante el proceso, ya que, dependiendo de los mecanismos y estructuras de gobernanza, la decisión per se recae en los tomadores de decisiones que normalmente escogemos en la etapa más básica de la participación procedimental, el voto.

El tercer requerimiento es el de contar con herramientas personales precisas para poder participar y es aquí donde ahora pondremos el foco. El primer requisito para una participación responsable y de peso es contar con la información necesaria para una correcta toma de decisiones. Básicamente se podría considerar el acceso, comprensión e internalización de la información, como un primer nivel de participación muy básico, ya que, si esta información no es utilizada para la toma de decisiones, no existe un verdadero nivel de incidencia. Se comienza a percibir como un proceso de participación más activo cuando se nos solicita o de manera voluntaria, utilizamos un espacio para emitir un criterio a partir de nuestras percepciones, conocimientos adquiridos y experiencias cotidianas. Por ejemplo, cuando emitimos nuestro voto, cuando somos parte de un proceso de consulta, validación o evaluación, entre otros. La tercera etapa se referiría entonces al momento en que tenemos una intervención activa, pudiendo ser esta el formar parte de un grupo organizado, creando y ejecutando ideas específicas, siendo parte de procesos de creación conjunta u otros similares; hasta llegar a altos niveles de participación como el ocupar un puesto de elección popular, o el formular y modificar normativa legislativa o política pública.

El participar en procesos del nombrado segundo y tercer nivel, sin contar con la información necesaria, provocaría un aumento en el riesgo de emitir criterios erróneos, sesgados, poco estratégicos o de impacto negativo o reducido, que pueden ser contraproducentes para el bien común.

Lo anterior nos lleva a las interrogantes de que, si todo ciudadano que está preparado para participar, tiene el deber de hacerlo, o si aquel que no está preparado o no tiene interés, debe participar de igual manera. Anduiza y Bosch (2004), resuelven estas interrogantes al aclarar que esto dependerá de si consideramos la participación como un fin en sí mismo o como un medio para conseguir un objetivo. Bajo el supuesto que ninguna persona está obligada a participar, podríamos decir que de cara al cambio climático lo ideal sería contar con una mezcla de ambos. Si bien es cierto que puede considerarse preferible que en la formulación de propuestas de mitigación y 
adaptación participen quienes más conocimiento técnico, experiencia práctica o más intereses tienen de aportar y/o aprender, podría ser un problema que algunos ciudadanos y ciudadanas mantengan una actitud apática y poco participativa.

Lo que no se puede dejar de lado aquí es que la inclusión de las distintas visiones y aportes, a partir de una participación más inclusiva pueden, por un lado, compensar en alguna medida las desigualdades sociales que podrían producirse en caso existan puntos de vista no tomados en cuenta y por otro, dinamizar los cambios que se necesitan para detener el aumento de la temperatura y adaptarse a las nuevas condiciones. En este sentido, según Anduiza y Bosch (2004), la dificultad más importante sería entonces la de cómo hacer efectiva la participación directa y continuada según corresponda.

\section{La gobernanza: ejercicio de la ciudadanía y las nuevas formas de participación}

Al engranaje entre la institucionalidad del Estado y la representación de los distintos sectores que lo componen le llamamos Gobernanza. En pocas palabras la gobernanza se podría definir como la interrelación de las estructuras formales e informales, así como los procedimientos, procesos y las formas de hacer cumplir las reglas, incluyendo a las redes de actores a todos los niveles de la sociedad.

La participación dentro de los sistemas de gobernanza es lo que podríamos llamar entonces como ejercicio de la ciudadanía. A pesar de que el término legal de ciudadanía se enmarca únicamente a una condición legal de mayoría de edad, que permite a la persona ejercer su derecho al voto como forma de participación procedimental, realmente el origen de este concepto se refiere más bien al interés y la posibilidad que tiene la persona (ciudadano-ciudadana) de incidir sobre los asuntos relacionados al bien común. Así, la ciudadanía se podría medir a un nivel político, entendiéndose este como el derecho a elegir y a ser elegido/a; civil, refiriéndose en este caso al pleno disfrute de las libertades individuales (expresión, asociación, etc.) y social, cuando se relaciona al derecho de tener una buena calidad de vida.

Considerando lo anterior, la ciudadanía podría aplicar entonces a toda persona que habita un lugar y va más allá del origen, estatus migratorios, orientación religiosa y/o condición legal de la mayoría de edad o estatus para emitir un voto o ser electo/a, y se relaciona más bien con el ejercicio pleno, consciente, activo y responsable de los deberes y derechos ciudadanos, como el de participación.
En contraposición al término de ciudadanía, en la antigua Grecia, se utilizaba el concepto de idiota, para referirse a aquella persona que se preocupa solo de sí misma, sin prestar atención a los asuntos públicos o políticos. En el contexto latinoamericano esta evolución entre el desinterés máximo, lo que es meramente el voto o la participación procedimental de la democracia (correspondiente a una expresión de una democracia representativa) y el ejercicio pleno de la democracia activa (o democracia participativa) -que se compone por los distintos niveles de participación-, está en un constante proceso de transformación. Sin embargo, en la última década se ha modificado, entre otras cosas, por un mercado hegemónico, compuesto por prácticas que podrían considerarse enajenantes e individualistas. Entre ellas, la virtualización de las relaciones humanas, que, en muchos casos, dificultan la percepción sobre lo que realmente está pasando a nuestro alrededor, permitiendo la invisibilización e inclusive la violentización de ciertos derechos humanos.

Siguiendo la teoría de Putman, anteriormente los distintos grupos de interés se involucraban en actividades más estructuradas, reguladas e institucionalizadas, con límites claros que demarcaban quiénes pertenecían y no pertenecían a las estructuras de gobernanza y a partir de ahí se consideraban buenos/as o malos/as ciudadanos/ as. En las últimas décadas, estas estructuras persisten, sin embargo, se observan estrategias de acción directa para temas específicos, comunicaciones virtuales, coaliciones temporales de estructuras planas y modos de pertenencia más informales, centrados en la preocupación e identidad común (Norris, 2002), los cuales no han sido del todo reconocidos y muchas veces no son percibidos como acciones de ciudadanía activa.

El fenómeno no se da únicamente en las juventudes, ni son estos los que necesariamente más participan en la Región; sin embargo hay que considerar que es probable que debido a que en algunas zonas de Latinoamérica los jóvenes se hayan socializado políticamente en un contexto de bienestar económico y social, es probable que en número, exista una mayor proporción tendiente a pensar en otros ámbitos posmaterialistas y estén más interesados en participar de actividades políticas más diversificadas y menos dirigidas por la élite política.

Con respecto a lo anterior Anduiza y Bosch (2004) separan la teoría "participativa" de la teoría "elitista", en dos grandes polos, utilizando como foco la forma de resolución del conflicto. Por un lado, para la teoría participativa, debe ser activa únicamente la ciudadanía interesada en la toma de decisiones, por lo que supone que todo aquel que no participa lo hace por insatisfacción o 
distanciamiento con la temática, dándole a una elevada participación la etiqueta de una mejor ciudadanía, mejor toma de decisiones y mejor ejecución de las mismas; sin embargo deja el vacío de cómo poner en marcha mecanismos masivos de participación de actores disímiles en muchos aspectos y algunas veces olvida que la población intensamente participante no es representativa del conjunto. Por otra parte, la teoría elitista, enmarca la participación únicamente en la elección de los representantes (democracia representativa) y deja la toma de decisiones únicamente a los políticos, por lo que la ausencia de participación en formas distintas al voto o la apatía participativa se entienden como satisfacción con el funcionamiento del sistema, ignorando así la importancia de los movimientos sociales como agentes de cambio.

Una combinación de ambas teorías permitiría el reconocimiento de las viejas y nuevas formas de participación como oportunidades de cara al cambio. Tomando como base a Putnam, podríamos decir que las generaciones más jóvenes se distancian de las formas de participación política más tradicionales como: el voto, la política electoral y partidista, y el asociacionismo más tradicional. Por otra parte, tomando a Norris de base, al parecer estas generaciones no necesariamente estarían dejando de participar, sino que podrían estar canalizando su accionar a través de otras nuevas acciones como la protesta.

Esta visión es apoyada por distintas realidades. En el caso europeo, por ejemplo, Morales $(2002,2005)$ observa que, a pesar de que la afiliación a partidos políticos ha disminuido, existe un creciente activismo político. Dentro de las formas de expresión de este activismo se ubica la participación en huelgas y manifestaciones, la firma de peticiones, e inclusive el asociacionismo o la conformación de ONG, que han crecido sustancialmente. Este mismo fenómeno parece suceder también en Latinoamérica. Sólo por mencionar algunos notables ejemplos planteados por la Encuesta Mundial de Valores (World Value Survey), dentro del contexto Latinoamericano se puede mencionar el caso de México, en que se presenta un aumento en la manifestación del activismo y la firma de peticiones, o el decline de estas mismas formas de participación en el caso Argentino. Otro caso reportado por el Guillén-Araya (2016) para el Estado de la Nación en Costa Rica, muestra un aumento en el número de manifestaciones, especialmente en temas relacionados al medio ambiente. Desde la participación formal y siguiendo con el caso costarricense, se presentan también mecanismos consultivos de carácter técnico y de sociedad civil, para la deliberación y el asesoramiento al Gobierno en torno a temas climáticos. Estas estructuras corresponden respectivamente al Consejo Científico de Cambio Climático (4C) y al Consejo Consultivo Ciudadano de Cambio Climático
(5C), los cuales están normados por Decreto y actualmente se encuentran en conformación, cuestión que anteriormente se hubiera categorizado como mecanismos de participación informal.

Según Anduiza y Bosch (2004), dentro de las formas de participación política se podrían citar, por un lado, aquellas Ilamadas como tradicionales, convencionales o formales, la mayoría de las veces normadas y en las que se ubica: el voto en elecciones o referéndum, la participación y colaboración en las campañas electorales, el ser miembro activo de un partido político u organización de carácter político, como los sindicatos, contactar con los medios de comunicación o con representantes políticos, colaborar en algún mecanismo de participación directa en políticas locales (como los consejos ciudadanos o los presupuestos participativos), entre otros. Y por otro lado, las emergentes, de protesta, no convencionales o hasta ahora informales en muchas latitudes, las cuales no utilizan los canales de participación institucionalizados y en ocasiones se refieren a acciones extralegales como: ser miembro activo de una ONG, grupo o asociación sobre cuestiones locales o globales, asistir a mítines, canvassing, participar en plataformas de escrutinio, financiar luchas específicas, pegar carteles o elaboración de grafitis, cargar distintivos de contenido político, participar en manifestaciones, huelgas u otras actos de protesta, insumisión o desobedecimiento de leyes, activismo cibernético, redes de política internacional, abandono de estructuras formales o boicot al consumo de determinados productos por razones políticas, éticas, medioambientales o su conjunto, lo que comúnmente se llama "consumo político".

Estas formas requieren distintos niveles de compromiso y cuentan con distintos grados de influencia, por ejemplo, según Norris (2002), votar es un fenómeno esporádico donde la presión en la toma de decisiones se ejerce sobre los líderes, pero de una manera difusa y con un amplio resultado que afecta a toda la ciudadanía; mientras que la participación en la formulación y aplicación de política pública requiere un compromiso más permanente, que requiere mayor iniciativa y esfuerzo que emitir un voto, y además necesita un mejor manejo de información.

Muchas de estas formas de participación provienen de la preocupación pública en torno a temas ambientales y de la necesidad de definir nuevos estilos de vida. Según Norris (2002), el ambientalismo presenta unos límites muy difusos ya que viene a acoger a grupos diversos que se encuentran con un tema en común, desde donde son afectados, por ejemplo: ecologistas, activistas por la paz, teóricos holísticos, activistas del poder, feministas, 
activistas de los derechos de los animales, antinucleares, el movimiento de la agricultura orgánica, el movimiento de la transición energética, los alimentos modificados genéticamente, entre otros.

El cambio climático, por su relación con la meteorología, se ha desarrollado desde la dimensión ambiental y ha trascendido muy lentamente hacia la temática del desarrollo integral, en la que se observan el resto de dimensiones. Debido a esta, aún débil relación, las formas de participación que más se han utilizado son las asociadas a los movimientos sociales, que basan su interés en dos vías, por una parte reformar el cuerpo normativo o influir en el proceso de política, y por otro alterar directamente los patrones sistemáticos del comportamiento social, incluyendo actividades no orientadas al estado sino hacia los consumidores, el sector privado e inclusive organizaciones internacionales, disminuyendo la autonomía del Estado y ejerciendo la presión de forma directa a otros grupos desde donde se piensa que debe surgir el cambio.

Así, desde el activismo o desde los movimientos sociales, algunas veces el accionar radica más en los cambios en el estilo de vida propio que en acciones que involucren formas de apoyo más orientadas a las políticas. Hago énfasis en que tradicionalmente el activismo ambiental guarda una relación negativa con la participación electoral y positiva con los movimientos de protesta (Norris, 2002). Aunque pareciera que en algunos casos, como el de Costa Rica, esta relación vaya variando, ambas vertientes de acción son importantes, y por lo tanto debe evitar dejar las formas de participación formal de lado.

\section{Requisitos para ser una parte responsable del proceso participativo}

Si bien es cierto que participar del proceso de toma de decisiones nos coloca en una posición con un alto nivel de responsabilidad, en el que muchas personas no desearían situarse, hoy en día parece necesario involucrarnos en mayor o menor medida para que podamos avanzar de una manera positiva y al ritmo que nos exige el cambio climático. Anteriormente los Gobiernos podían darse el lujo de ser paternalistas y tomar decisiones sin consultarnos, debido a que la población era menor y los niveles educativos eran normalmente más bajos; sin embargo, el aumento de la población y la necesidad de considerar a la pluralidad que la distingue, dentro de la toma de decisiones, requiere al menos, de la representación de sus distintos sectores.

Otro importante punto a considerar por parte de los Gobiernos, es que una participación motivada por el interés tiene una interpretación y unas consecuencias muy distintas a una participación motivada por el descontento (Anduiza \& Bosch, 2004). La participación puede estar motivada por actitudes de rechazo al sistema político o insatisfacción con el funcionamiento del mismo, lo que cambia la interpretación del fenómeno y el tipo de accionar. Viéndolo desde el punto de vista institucional, la aceptación y promoción de vías formales de participación, así como la consecución de sus decisiones, fomenta el cumplimiento voluntario de la ley y la capacidad de los gobiernos para implementar legislación efectiva, al mismo tiempo que promueve la consolidación en las democracias más nuevas; lo cual representaría una opción para los Gobiernos de mejorar su imagen política. Actualmente es ampliamente percibido que muchos Estados funcionan más a la merced del mercado que al del bien común, provocando que un grueso de las poblaciones haya comenzado a dudar por un lado, de las capacidades y motivaciones del Estado, y por otro, de las intenciones del mercado, en el que décadas atrás confiaban poderosamente. Este panorama ha provocado, entre otras cosas, una "privatización" o "liberalización" de muchos Estados, es decir, la disminución del grado de influencia del Estado en asuntos estatales, a causa de un aumento de la influencia del mercado en las mismas; lo cual trae como consecuencia un mal manejo de los bienes y servicios públicos, y una imagen negativa por parte de la ciudadanía, que pone en el Gobierno el cuido de lo público.

Paralelo a esto, en este nuevo panorama donde se generan nuevas pautas de participación, también se abren las puertas a dos sectores con mucho poder, que históricamente habían perdido fuerza, pero que poco a poco, a raíz de este cambio en el interés de lo público, la han ido recuperando. Estos son la ciencia y la religión, bajo las cuales, los colectivos se están apoyando cada vez más como herramientas para dirigir la toma de decisiones y confiar en el proceso, sirviendo muchas veces como opciones alternativas al Gobierno. Ambos sectores, tienen en este momento una gran oportunidad de fortalecer su imagen y presencia en la ciudadanía. Qué sucederá en la evolución del poder de estos nuevos actores y qué pueda significar esto para el bien común en un contexto de cambio climático, dependerá del nivel, seriedad y profundización con que las personas se involucren y demanden su accionar.

Es por esto que, si bien es cierto que, tal y como menciona Torres-Rivas (2001), esta profundización en los niveles de democracia, implica "descansar la fuerza de la democracia no sólo en la unanimidad o el consenso sino en el pluralismo del conflicto", el recambio generacional en cuanto a la nueva pluralidad en niveles y formas de 
participación política, no significa que las nuevas formas vayan a ser mejores, más asertivas o más estratégicas, ni tampoco indica que estas formas vayan a facilitar el desarrollo de ciudadanos más participativos o con intensiones mejor dirigidas.

Operativamente hablando, lo anterior plantea interrogantes de ¿cómo considerar los diversos intereses desde la institucionalidad democrática y evitar al mismo tiempo la creación de poderes paralelos al Gobierno? La respuesta parece ser clara y se refiere a la creación de sinergia entre el Estado y la ciudadanía, bajo un enfoque solidario e intergeneracional, en donde los intereses a considerar en el proceso de toma de decisiones deben ser los intereses públicos, no los sectoriales y mucho menos los individuales.

En este sentido debe entenderse por interés público, a aquellos asuntos en disputa que afectan propiedad pública o donde están en juego derechos y/o intereses colectivos. En otras palabras, un interés público debe hacer referencia a los bienes y servicios que no pertenecen a una persona específica, y que, por el contrario pertenecen al conjunto, a todas y todos, incluyendo en esta lista, a las próximas generaciones.

Esta necesaria consideración de las pluralidades ante un escenario de cambio climático implica por tanto, escoger de la mejor forma, a nuestros y nuestras representantes, siendo una posibilidad el ofrecernos para representar, si es que realmente contamos con las capacidades para hacerlo. Por otra parte, implica, además, un diálogo constructor de acuerdos, incluir y considerar las distintas perspectivas de la pluralidad (ojalá informadas) y una negociación en búsqueda de un ganar-ganar de las partes involucradas.

Para que el diálogo sea exitoso y la negociación dé frutos que generen impactos positivos, es necesario primeramente, que cada parte tenga claros sus intereses. Es decir, que las partes conozcan los fines del resultado que esperan del proceso. En otras palabras, que sepan el para qué, y no solamente el qué es lo que desean. Conocer el para qué de una negociación, el interés principal de ésta, abre el abanico de opciones estratégicas que pueden lograr el impacto buscado, mientras que basar una negociación en el qué, reduce el impacto deseado a una única posibilidad de alcanzarlo.

En esta misma línea, otro requisito del diálogo constructor de acuerdos, es que las partes sepan diferenciar entre sus necesidades y sus demandas. Esto es importante porque la demanda per se podría convertirse en el qué se desea, mientras que la necesidad de fondo podría equivaler al para qué, abriendo una vez más el abanico de oportunidades para la negociación.
Un tercer punto a considerar en el diálogo es que las partes brinden y sepan compartir espacios y oportunidades, permitiendo evitar que una de las partes pierda en el proceso, o peor aún, se desaproveche el espacio al que se fue invitado, provocando que no se le invite más o que su invitación se vuelva más bien contraproducente para los resultados esperados. Por último, y como se ha mencionado anteriormente, es importante que al ser parte del diálogo y la negociación, se haga lo posible por disminuir las brechas de acceso y manejo de información, de modo que cada parte pueda velar porque los acuerdos tomados no vayan a perjudicarle.

Otros puntos básicos a considerar cuando participamos de procesos de toma de decisión, incluyen contar con un discurso sólido y bien fundamentado, saber que es permitido disentir y que alguien puede pensar diferente al conjunto, contar con una representación diversa y que esta esté incluida dentro del discurso y de la práctica, desarrollar una estrategia de comunicación basada en cómo los demás escuchan, y por supuesto, no agotar a las personas, ya que esto, además de egoísta, podría producir una pérdida de interés y por lo tanto de apoyo, en el proceso.

\section{Oportunidades para la incidencia de asuntos ambientales y relacionados al cambio climático}

Hablando específicamente de bienes y servicios públicos relacionados al cambio climático y en los que la ciudadanía debería tratar de incidir en la toma de decisiones, en búsqueda de un bien común, se podrían citar a manera de sencillos ejemplos: la diversidad de ecosistemas y con ellos los bienes y servicios que podrían ser alterados con el cambio del patrón climático, las fuentes de agua, las tierras fértiles, las nubes, la lluvia, entre otros, los cuales podrían estar en riesgo para una población importante del planeta, de no tomarse las medidas correctas en un corto plazo. Nos encontramos en una época que se enfrenta a nuevos patrones del clima, a nuevas realidades en cuanto al acceso y distribución de bienes y servicios ecosistémicos, y por tanto, a una mayor vulnerabilidad.

En otras palabras, siendo el cambio climático, una de las mayores fuentes de conflictos socio-ambientales del antropoceno, se convierte en la perfecta oportunidad para acercarnos un poco más a la justicia social, la sostenibilidad ambiental, el desarrollo económico inclusivo y por tanto, a una transformación social y ecológica que permita una mayor calidad de vida para esta y las próximas generaciones. Tomar decisiones podrá ser tarea del Estado, pero elegir a nuestros y nuestras representantes, 
generar y brindar insumos para su toma de decisiones, velar porque nuestros intereses en búsqueda del bien común sean representados e incomodarnos un poco en nuestra forma de vida, es también nuestra tarea y esta sólo se logrará si somos parte del proceso de cambio.

Específicamente en temas de adaptación, el trabajo local parece representar una opción más sencilla de búsqueda de equilibrio entre el crecimiento económico y la sostenibilidad ambiental y la justicia social, sin embargo temas como la disminución de gases de efecto invernadero pareciera que debieran tratarse más bien de forma transnacional y bajo otro tipo de alianzas.

Con el fin de cumplir con los compromisos climáticos del Acuerdo de París y de cara al año 2020, en el que los países deben presentar la renovación de sus compromisos ambientales, y entran en vigor muchos acuerdos en el marco de la diplomacia ambiental, existe una serie de oportunidades para reforzar nuevos mecanismos de participación ciudadana. Si tomamos como base el propio Acuerdo de País, los artículos 12 y 13 nos reflejan algunas de estas oportunidades:

Artículo 12: "Las Partes deberán cooperar en la adopción de las medidas que correspondan para mejorar la educación, la formación, la sensibilización y participación del público y el acceso público a la información sobre el cambio climático, teniendo presente la importancia de estas medidas para mejorar la acción en el marco del presente Acuerdo."

Artículo 13: ... "el propósito del marco de transparencia de las medidas es dar una visión clara de las medidas adoptadas para hacer frente al cambio climático"...

Con esto se extrae que, desde el punto de vista institucional y de participación formal, durante estos años los Estados deben generar o reforzar mecanismos de coordinación institucional y apoyo a la Contribución de Acciones Determinadas (NDC), pero además de esto deben decidir sus necesidades en materia de financiamiento, tecnología y creación o fortalecimiento de capacidades; así como reforzar sus mecanismos de Medición, Reporte y Verificación, inventarios de Gases de Efecto Invernadero, políticas de Gobierno Abierto, entre otros. Por su parte la ciudadanía debe utilizar las mejores herramientas y estrategias para demandar la transparencia y la apertura de espacios, así como participar activa, informada y responsablemente (Granados-Solís, 2017).

\section{Conclusiones y recomendaciones prácticas para promover la participación ciudadana ante el cambio climático}

A pesar de que la nueva ciudadanía posee prácticas que podrían considerarse enajenantes e individualistas, las formas de ejercer ciudadanía y participar de los procesos de toma de decisiones, pueden ser muy diversas e incluyen tanto opciones formales como no formales, propositivas, como contestarías, con foco en alterar directamente los patrones sistemáticos del comportamiento social o de influir en el proceso de política pública; todas ellas en distintos niveles de profundidad y con distintas posibilidades de incidencia. Por lo tanto, pensar que nos encontramos en una crisis de participación podría ser incorrecto; lo que si debemos cuestionarnos es si las nuevas pautas de participación, como ejercicio de la ciudadanía para un bien común, son las más eficientes para el propósito y si la velocidad de injerencia es suficiente para enfrentar los nuevos escenarios producto del cambio climático.

En definitiva tanto las acciones formales como no formales, son necesarias y deben permanecer en el abanico de opciones, sin embargo es necesario, por un lado, cuestionarnos si el momento, la intensidad y la frecuencia con la que las utilizamos es la más acertada y por otro, trabajar sobre ciertos retos para que estas acciones sean realmente eficientes y dirigidas a la sostenibilidad ambiental y justicia social, que podría verse aún más en riesgo con este fenómeno. Entre estos retos están el robustecer las habilidades ciudadanas para que la democracia sea realmente activa, y fortalecer las capacidades del aparato institucional y su cuerpo normativo, de forma que sea posible brindar espacios para la participación, asegurándose de que esta sea legitimada. Además, debemos tener claros nuestros intereses y su relación con el bien común, estar organizados, incluir a la pluralidad en la discusión, combatir los intereses no fundamentados con conocimiento, contar con una visión estratégica en la que se tenga claro que, aunque no veamos la meta a la que queremos llegar, somos parte del proceso y por tanto, debemos crear y aprovechar nuestras propias oportunidades. Por último, internalizar el hecho de que el conflicto que nos produce el cambio climático, no es necesariamente un problema y que dependiendo de cómo lo manejemos, este conflicto más bien podría representar una oportunidad de solventar todo aquello 
que históricamente nos ha dividido, nos ha generado roces o nos puede perjudicar, a unos más a largo plazo que a otros.

La forma menos riesgosa, menos costosa y más productiva para alcanzar el bien común, en esta época en la que nos enfrentamos a una crisis de magnitud global como lo es el cambio climático, es a través del ejercicio de la ciudadanía activa y responsable, desde lo que cada ciudadano y ciudadana pueda aportar. El cambio climático viene a ser un espacio de lucha con muy diversos propósitos, más relacionados con el desarrollo y la calidad de vida, que con asuntos meramente de corte ecológico o ambiental. Las posibilidades de que los distintos grupos y las distintas luchas encuentren cabida en esta gran sombrilla es bastante grande y por ende, el aporte que pueda hacer la pluralidad hacia un cambio más positivo es también de suma importancia. En este sentido, la participación debe darse con miras a incluir las perspectivas de las pluralidades y en aras de velar por lo público, lo cual puede significar el cuido de los derechos humanos y la no privatización de ciertos bienes y servicios ecosistémicos.

Debido a que los Gobiernos y los mercados han perdido muchas fuerzas, actores ligados a la ciencia o a la religión, podrían tener en este espacio un amplio espectro de acción y capilaridad para fomentar una correcta y responsable participación. En caso de que los Gobiernos y los mercados deseen contar nuevamente con el grueso de la ciudadanía, como lo hicieron en épocas pasadas, deberán dirigir su accionar hacia la defensa de lo público, agregando variables de calidad de vida y no sólo de crecimiento económico.

\section{REFERENCIAS}

Anduiza, E., \& Bosch, A. (2004). Comportamiento político y electoral. Barcelona: Ariel.

Barnes, S. y Kaase, M. (1979). Political Action: Mass Participation in Five Western Democracies. Beverly Hills: Sage.

CEPAL (Comisión Económica para América Latina y el Caribe). (2018). Acceso a la información, la participación ylajusticia en asuntos ambientales en América Latina y el Caribe: hacia el logro de la Agenda 2030 para el Desarrollo Sostenible (LC/TS.2017/83). Recuperado de: https://repositorio.cepal.org/bitstream/handle/11362/43301/4/ S1701021_es.pdf

Comisión de Derechos Humanos. (1948). Declaración Universal de los Derechos Humanos. Naciones Unidas, Francia.

Granados-Solís, G. (2017). La relevancia de la transparencia, apertura y participación ciudadana en la lucha contra el cambio climático. Sinergias, 25-28.
Guillén-Araya, J. M. (2016). El Panorama de las Acciones Colectivas en Costa Rica. Vigesimosegundo Informe Estado de la Nación en Desarrollo Humano Sostenible, Costa Rica.

Guzmán, S., \& Castillo, M. (2016). INDCs y participación ciudadana en América Latina. Grupo de Financiamiento Climático para América Latina y el Caribe (GFLAC). Recuperado de: http://extrayendotransparencia.grupofaro.org/wp-content/uploads/2015/12/292797341-INDCs-y-participacion-ciudadana-en-America-Latina.pdf

Hernández Valle, R. (2002). De la democracia representativa a la democracia participativa. Anuario Iberoamericano de Justicia Constitucional, 6, 199-219 pp. Recuperado de: https://recyt.fecyt.es/index.php/AIJC/article/ view/50596/30832

Milbrath, L. y Goel, M. (1977). Political participation: how and why do people get involved in politics?. Chicago: Rand McNally Colleg.

Morales, L. (2002). Associational Membership and Social Capital in Comparative Perspective: The Problems of Measurement. Politics and Society, 30, 497-523.

Morales, L. (2005). ¿Existe una crisis participativa? La evolución de la participación política y el asociacionismo en España. Revista Española de Ciencia Política, 13, 51-87. Recuperado de: https://recyt.fecyt.es/index.php/recp/ article/view/37411

Norris, P. (1999a). Conclusions: The Growth of Critical Citizens and Its Consequences, en Pippa Norris, ed., Critical Citizens. Oxford: Oxford University Press.

Norris, P. (1999b). Critical Citizens. Global Support for Democratic Governance. Nueva York: Oxford University Press.

Norris, P. (2002). Democratic Phoenix. Reinventing Political Activism. Cambridge: Cambridge University Press.

Plataforma LEDS LAC et al. (2017). Mecanismos de Coordinación Interinstitucional para una Política Climática Efectiva en Latinoamérica y el Caribe Documento de trabajo. Grupo Banco Mundial. Recuperado de: http:// ledslac.org/wp-content/uploads/2017/03/ Mecanismos-Coordinaci\%C3\%B3n-Interinstitucional.Working-Paper_final.pdf

Putnam, R, \& Goss, K. A. (2002). Introduction, en Robert Putnam, ed., Democracies in Flux. The Evolution of Social Capital in Contemporary Societies. Nueva York: Oxford University Press.

Torres-Rivas, E. (2001). Contrapunto entre reforma y revolución: La democracia en Costa Rica y Guatemala. Trabajo elaborado durante la estadía de investigación en el David Rockefeller Center for Latin American Studies de la Universidad de Harvard durante el segundo trimestre del año 2000. Recuperado de: http://bibliotecavirtual. clacso.org.ar/ar/libros/coedicion/rivas/06contra.pdf

Welzel, C., Inglehart, R., \& Deutsch, F. (2005). Social Capital, Voluntary Associations, and Collective Action: Which Aspects of Social Capital Have the Greatest "Civic" Payoff? Journal of Civil Society, 1(2), 121-146. 